\title{
CdSe nanoparticles grown with different chelates
}

\author{
Yu.Yu. Bacherikov ${ }^{*}$, M.O. Davydenko², A.M. Dmytruk ${ }^{3}$, I.M. Dmitruk ${ }^{2}$, P.M. Lytvyn ${ }^{1}$, \\ I.V. Prokopenko ${ }^{1}$, V.R. Romanyuk ${ }^{1}$ \\ ${ }^{I} V$. Lashkaryov Institute of Semiconductor Physics, NAS of Ukraine, 41, prospect Nauky, 03028 Kyiv, Ukraine \\ *E-mail: yuyu@isp.kiev.ua \\ ${ }^{2}$ Taras Shevchenko Kyiv National University, Physics Department, 2, Build 1, prospect Academician Glushkov, \\ 03127 Kyiv, Ukraine \\ ${ }^{3}$ Center for Interdisciplinary Research, Tohoku University, Sendai, 980-8578, Japan
}

\begin{abstract}
Modified reverse micelles method allowing fabrication of CdSe nanoparticles in toluene solution in series of sizes with average diameter from 1.2 to $3.2 \mathrm{~nm}$ and size distribution $\sim 12-30 \%$ is presented. Simple empirical relation between the CdSe nanoparticle diameter and exciton absorption wavelength is proposed, which allows to do prompt and effective monitoring the particles size and size distribution during the synthesis. Optical absorption and photoluminescence measurements as well as EDX demonstrated good quality of obtained nanocrystallites. Besides, study of nanoparticles produced using two complexing agents (SNTA and Trilon B) revealed similar stoichiometric and optical properties. Trilon B is suitable for CdSe nanoparticles growth instead of SNTA. Because of higher stability of the chelate complex of Trilon B and $\mathrm{Cd}^{2+}$ ions, it is possible to use higher temperature for growth which allows preparation of large size nanocrystals.
\end{abstract}

Keywords: CdSe, nanoparticle, quantum size effect, chelating agent, stoichiometry.

Manuscript received 23.02.06; accepted for publication 29.03.06.

\section{Introduction}

Interest to nanoscale matter is so high that every possible method of their production is tried out. It is caused by a variety of new physical properties of nanosized particles comparing to bulk materials with the same chemical constituents. Therefore, some properties of materials can be altered without any changes in chemical composition. Modern development of science and technology brings to the foreground the creation of ordered nanosized structures and new devices based on them.

Another important aspect is synthesis of quantum dots with a narrow size distribution, because this factor determines size-sensitive electrical, magnetic and optical properties of obtained materials. Wet chemical methods are suitable for synthesis of nanoparticles with different sizes and shapes [1], also they are perspective for production of large amount of material. Wet chemical methods for synthesis of nanoparticles give us an opportunity to obtain the particles in solution with a necessary size by varying, for example, the growth time and temperature. From the other point of view, chemical methods are relatively cheap and simple in comparison with the methods such as laser ablation, molecular-beam epitaxy or electron beam lithography. But besides the advantages there are also some imperfections such as production safety, environment pollution and problems with waste utilization.

The most interesting for scientists are metal and $\mathrm{A}^{2} \mathrm{~B}^{6}$ compound semiconductor nanoparticles which found applications in different areas. That is why progress in study of properties and methods of synthesis of these nanosized materials is observed. At present, a few chemical methods for CdSe nanoparticle synthesis are widely used [2-6]. The method proposed by Murray et al. [2] is currently the most popular for commercial mass production of $\mathrm{CdSe}$ nanoparticles. But this method requires usage of high temperatures and airless procedures, which makes it complicated. Nevertheless it allows obtaining the CdSe nanocrystallites with sizes up to $\sim 11 \mathrm{~nm}$ and size distribution $\sim 5 \%$ with additional facilities such as a size-selective precipitation or special processing equipment [7].

Kasuya et al. [3] exploited the ability of surfaceactive-agent (surfactant) to self-organize into micelles (or reverse micelles) [8]. The volume confined by 
micelles serves as a template and limiting factor during nanoparticles growth [9].

As a source of $\mathrm{Cd}^{2+}$ ions, the different compounds can be used, such as dimethylcadmium [2], $\mathrm{CdSO}_{4}$ [3], $\mathrm{CdCl}_{2}[4,5], \mathrm{Cd}\left(\mathrm{CH}_{3} \mathrm{COO}\right)_{2}$ [6]. Slow release of free metal cations in the course of nucleation and growth of nanoparticles is an important aspect of the synthesis reaction. It must be considered in choosing the metal chelating agent, because it influences on reaction dynamics, temperature regime, and, accordingly, on the size and polydispersity of obtained nanoparticles. Different surfactants and metal chelating agents such as tri-n-octylphosphine and tri-n-octylphosphine oxide (TOP, TOPO) [2], decylamine $\left(\mathrm{CH}_{3}\left(\mathrm{CH}_{2}\right)_{9} \mathrm{NH}_{2}\right)$ [3], sodium mercaptoacetate [4], gelatin [4], cetyltrimethylammonium bromide (CTAB) [5] are used for this purpose.

Therefore, in this paper we present the results of investigations of the effect inherent to two chelating (complexing) agent - nitrilotriacetic acid disodium salt (SNTA) and ethylenediaminetetraacetic acid (EDTA) disodium salt (Trilon B) that is pronounced in the growth of CdSe nanoparticles in colloidal solution by the wet chemical method. In general, the method described in [3] was used as a basis.

Characterization of prepared nanoparticles was performed by atomic-force microscopy (AFM), energy dispersive X-ray spectroscopy (EDX), optical absorption and photoluminescence (PL) methods.

\section{Nanoparticles preparation and measurement techniques}

CdSe nanoparticles were prepared by the wet chemical method in reverse micelles in toluene. As a source of $\mathrm{Se}^{2-}$ ions, sodium selenosulphate $\left(\mathrm{Na}_{2} \mathrm{SeSO}_{3}\right)$ (solution 1) was used. Solution 1 was prepared by the vigorous stirring of $\mathrm{Se}(99+\%)$ with $\mathrm{Na}_{2} \mathrm{SO}_{3}$ in distilled water for about two days at $70{ }^{\circ} \mathrm{C}[10] . \mathrm{Cd}^{2+}$ ions source was cadmium nitrilotriacetate (solution 2). Solution 2 obtained by dissolving the $\mathrm{CdSO}_{4}$ and complexing agent in water and with decylamine $\mathrm{CH}_{3}\left(\mathrm{CH}_{2}\right)_{9} \mathrm{NH}_{2}$ as surfactant. The amine groups of the surfactant and chelating agent molecules form complexes with metal ions, which prevents $\mathrm{Cd}^{2+}$ from oxidation in water solution as well as fast uncontrolled reaction with $\mathrm{Se}^{2-}$ ions. These complexes may slowly dissociate at high $\mathrm{pH}$ $(\mathrm{pH} \approx 11.5$ in our case) or high temperature. Authors in [3] used SNTA as a chelating agent (method 1). In this work, for cadmium ions binding common and cheaper chelating agent - Trilon B (method 2) was used. Solutions 1 and 2 are mixed with the following injection of toluene. When adding toluene to this solution, the micelles move up into the toluene and transform into reverse micelles in which nucleation and further nanoparticle growth takes place. Within a few minutes, the toluene turns uniformly to greenish yellow, whereas the water phase remains colourless. As reported in [3], in the solution prepared at the near-room temperature the so-called magic nanoclusters $\mathrm{CdSe}_{33}$ and $\mathrm{CdSe}_{34}$ prevail demonstrating a remarkable narrow size distribution on atomic scale.

Further nanoparticle growth takes place at the elevated temperature above $45{ }^{\circ} \mathrm{C}$. Particle growth stops as soon as heating was switched off. It allows obtaining the nanoparticles of different sizes by varying only the synthesis temperature and time (up to several hours). A series of pair of samples were produced according to "method 1" and "method 2" for different time $(t=$ $=30 \ldots 120 \mathrm{~min})$ and temperatures $\left(T=90 \ldots 130{ }^{\circ} \mathrm{C}\right)$ only with a difference in metal chelating agent used.

The AFM (NanoScope IIIa, DI, USA) tapping mode was applied for particle size measurements. Standard silicon probes with nominal tip radius of $10 \mathrm{~nm}$ (NT-MDT, Russia) were used. Because of the tip effect, vertical sizes of studied particles are taken into account. For AFM study, dispersed nanoparticles were deposited on the fresh cleavage of mica substrate.

Scanning electron microscope (SEM) (Hitachi S4300) equipped with the energy disperse X-ray analyzer (EDX) was utilized to study the composition of prepared $\mathrm{CdSe}$ nanoparticles. Accelerating voltage was $10 \mathrm{kV}$, which is high enough to provide reliable detection of $\mathrm{Cd}$ and Se and sufficiently low to prevent electron beam penetration to a substrate (highly oriented pyrolytic graphite, HOPG) used in the measurements.

Optical measurements were performed at the room temperature with the setup based on the monochromator MDR-3. For optical measurements, the colloidal solution of CdSe nanoparticles was placed into the quartz cylinder cell with the diameter $10 \mathrm{~mm}$. Optical absorption measurements were referenced against toluene. Photoluminescence spectra were measured at $90^{\circ}$ geometry with nitrogen laser excitation $(\lambda=$ $=337.1 \mathrm{~nm}$, the pulse repetition rate $50 \mathrm{~Hz}$, the average power $3 \mathrm{~mW}$ ).

\section{Experimental results and discussion}

\subsection{AFM measurements of characteristic sizes of nanoparticles}

AFM images of deposited on the mica substrate nanoparticles of samples \#1 (prepared according to "method 1", i.e. with SNTA) and sample \#2 (prepared according to "method 2", i.e. with Trilon B) are presented in Fig. 1. As it can be seen from the AFM pictures, the size distribution of obtained nanoobjects is quite narrow, that is why it can be concluded that the nanopowder is well dispersed and the majority of nanoformations corresponds to single nanoparticles. The sample concentration was chosen in such a way that the nanoparticles formed a monolayer on the substrate and the distance between particles was sufficiently large for their separation by AFM probe. Due to such a spacing, the vertical size values were not distorted by the finite size of the AFM probe. 


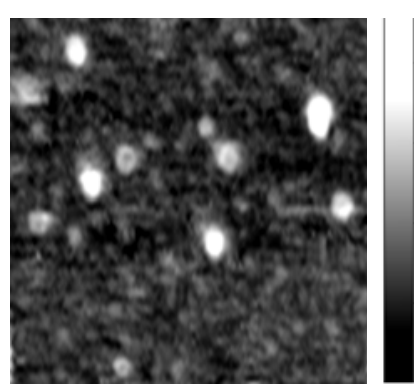

$a$

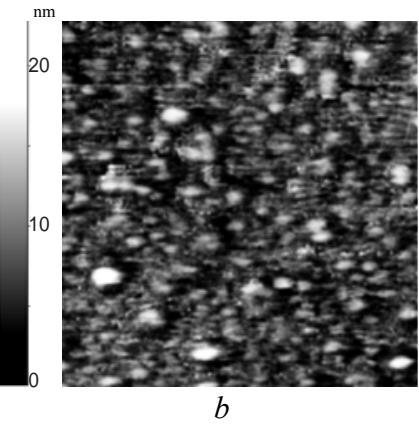

Fig. 1. AFM images of CdSe nanoparticles on the mica substrate of the sample \#1 (a) and the sample \#2 (b). Scan size $500 \times 500 \mathrm{~nm}^{2}$.

Vertical sizes were estimated from the crosssections of AFM images (Fig. 2). Each cross-section was made along the particle diameter. The average vertical size of particles of the sample \#1 is $2.0 \pm 0.5 \mathrm{~nm}$, and for the sample $\# 2-1.50 \pm 0.25 \mathrm{~nm}$.

\subsection{EDX measurements}

EDX measurements were made to analyze the chemical composition of obtained nanoparticles. Stoichiometry is an important property for light-emitting nanoparticles.

For the sample \#1, the Cd:Se ratio is $0.96 \pm 0.15$, for the sample \#2 - $0.77 \pm 0.09$. The concentration of chelating agent influences on the rate of $\mathrm{CdSe}$ nanoparticle formation. This chemical reaction can be accelerated or decelerated by adding different quantities of the chelating agent. We could obtain $\mathrm{CdSe}$ nanoparticles with the $\mathrm{Cd}$ :Se ratio in the range of 0.77 to 1.1 by varying only the Trilon B concentration.

Also, the analysis of the EDX spectra has shown a presence of $\mathrm{C}$ atoms originating, obviously, from the organic shell of nanoparticles.

Thus, the EDX elemental analysis confirms successful preparation of CdSe nanoparticles by both methods. Basing on the data [2,3], it is worth to note that $\mathrm{CdSe}$ nanoparticles with the sizes larger than $2 \mathrm{~nm}$ have a crystal-like (predominantly zinc-blende) structure, i.e. they are nanocrystallites.

\subsection{Optical absorption and photoluminescence}

Absorption spectra for series of samples with different sizes produced with SNTA and Trilon B are presented in Fig. 3. Every spectrum has its characteristic absorption peak that shifts continuously from 480 to $560 \mathrm{~nm}$ depending on the size of nanoparticles.

The dependence of the energy of electron transitions between quantized levels of the valence and conduction bands on the particle size was used to estimate the average particle size. Such transition is often called excitonic [11], because an electron-hole pair generated by light absorption is similar to the WannierMott exciton in a bulk crystal. In this paper, attention is paid to the particles with average sizes $d=1.5 \ldots 3.5 \mathrm{~nm}$, for which the exciton Bohr radius $\left(a_{\mathrm{B}}=5.6 \mathrm{~nm} \mathrm{[10])}\right.$
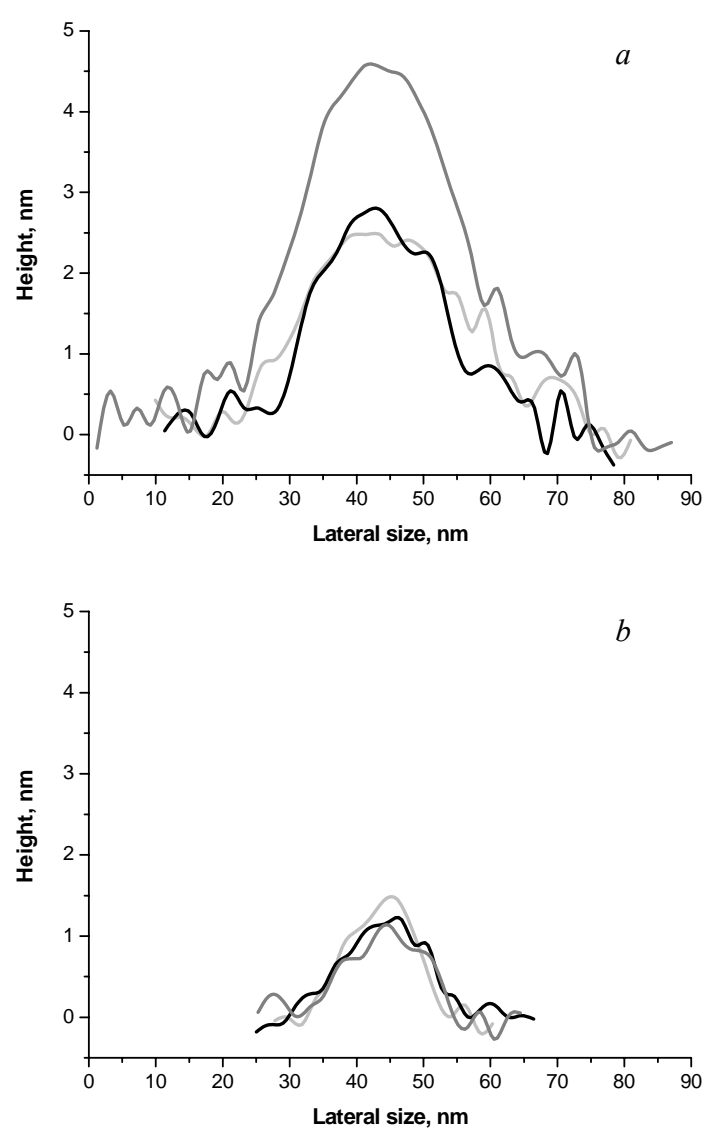

Fig. 2. Typical vertical cross-sections of CdSe nanoparticles of the sample \#1 (a) and sample \#2 (b). Cross-sections are plotted along the diameters of the particles.

exceeds a particle size. That is why for these particles a strong confinement is realized [12].

The simplest model based on the effective-mass approximation predicts the exciton energy shift, $\Delta E$, due to three-dimensional confinement comparing to the bulk one as [13]:

$$
\Delta E=\frac{\hbar^{2} \pi^{2}}{2 R^{2}}\left(\frac{1}{m_{e}}+\frac{1}{m_{h}}\right)-1.786 \frac{e^{2}}{\varepsilon_{\infty} R}-0.248 E_{\mathrm{Ry}}^{*},
$$

where $R=d / 2$ is the particle radius, $m_{e}$ and $m_{h}$ are the electron and hole effective masses, $\varepsilon_{\infty}$ is the dielectric constant, and $E_{\mathrm{Ry}}^{*}$ is the effective Rydberg energy $e^{4} / 2 \varepsilon^{2} \hbar^{2}\left(m_{e}^{-1}+m_{h}^{-1}\right)$. The first term in Eq. (1) represents the kinetic energy, the second term - the Coulomb one, and the third is a result of the correlation effect. But exciton energies calculated with this model somehow do not agree with the experimental values, especially for smallest particles.

A better description of the band structure has been obtained within the tight-binding calculations [13, 14]. The agreement with the experimental data is quite well for nanoparticle sizes larger than $\sim 2 \mathrm{~nm}$. Below $2 \mathrm{~nm}$, the tight-binding calculation starts to deviate essentially from the experimental values.

\section{(C) 2006, V. Lashkaryov Institute of Semiconductor Physics, National Academy of Sciences of Ukraine}




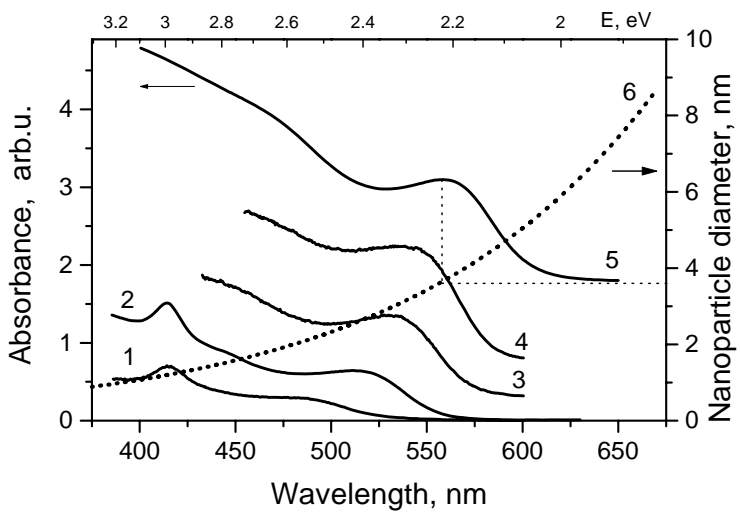

Fig. 3. Room temperature absorption spectra of $\mathrm{CdSe}$ nanoparticles synthesized in solution for different time and temperatures of growth: curve $1-$ sample \#3 $(t=30 \mathrm{~min}, T=$ $\left.=80{ }^{\circ} \mathrm{C}\right), 2-$ sample $\# 4\left(30 \mathrm{~min}, 100^{\circ} \mathrm{C}\right), 3-$ sample $\# 5$ $\left(60 \mathrm{~min}, 100^{\circ} \mathrm{C}\right), 4$ - sample \#6 $\left(120 \mathrm{~min}, 100^{\circ} \mathrm{C}\right), 5$ sample $\# 7$ (180 $\left.\mathrm{min}, 110^{\circ} \mathrm{C}\right)$. The samples represented by curves 2 and 5 were prepared with SNTA, curves 1,3 and $4-$ Trilon B. Dotted curve 6 presents calculated according to Eq. (2) dependence of CdSe nanoparticles diameter on exciton peak wavelength.

That is why, it would be more expedient to work out the known for CdSe empirical dependence of $E(d)$ for most precise estimation of the particle size from optical absorption spectra. Experimental data of the energy position of the absorption peak for $\mathrm{CdSe}$ nanoparticles of different sizes were presented in many papers. The most reliable are the results given in [2]. Murray et al. used high resolution transmission electron microscopy and performed statistical processing the obtained data for a wide range of particle sizes. It allows us to build the dependence of the exciton energy on diameters of CdSe particles. For the studied size range, it is convenient to plot the dependence of the wavelength of the absorption peak $v s$ the logarithm of the number of atoms in the nanoparticle. It was found that the dependence is linear, and it can be approximated by the least-squares method. It results in the simple empirical relation between the $\mathrm{CdSe}$ nanoparticle diameter and exciton peak position:

$d[\mathrm{~nm}]=0.344 \exp \left(\frac{\lambda_{\max }[\mathrm{nm}]-252.7}{129.3}\right)$.

Check of its precision shows that the error in diameter estimation for the smallest particles with the sizes $1.2 \ldots 3.2 \mathrm{~nm}$ is smaller than $0.1 \mathrm{~nm}$ and for larger particles $-0.2 \ldots 0.3 \mathrm{~nm}$. In this paper, we used Eq. (2) to estimate the average diameter of $\mathrm{CdSe}$ nanoparticles grown in solution as shown in Fig. 3 (curve 6). This relation is valid for nanoparticles within the size range up to $\sim 10 \mathrm{~nm}$, when $d>a_{\mathrm{B}}$ and exciton energy shift due to three-dimensional confinement vanish.

So, as it can be seen from Fig. 3 the largest particles obtained with usage of SNTA have the average diameter $3.7 \mathrm{~nm}$ with usage of Trilon $\mathrm{B}-3.2 \mathrm{~nm}$. But as stability of chelating agent and metal cation complex at elevated temperature is higher for Trilon B $[15,16]$, it makes possible usage of this method (when its modification is non-significant) for obtaining the nanoparticles with larger sizes.

The dispersion of nanoparticle sizes $\Delta d / d$ in an ensemble can be estimated from the full width at half maximum (FWHM) of the absorption peak. This method is based on the fact that inhomogeneous broadening is caused mainly by size distribution of nanoparticles in solution. Homogeneous broadening is determined from the experiments of photoetching [17] as $23 \mathrm{~nm}$ and is excluded from the measured FWHM.

According to the procedure described above, the average size of particles in the sample \#5 is found as $3.0 \mathrm{~nm}, \Delta d / d=19 \%$; in the sample \#6 - $3.2 \mathrm{~nm}$ and $20 \%$; in the sample \#7 $-3.7 \mathrm{~nm}$ and $22 \%$, respectively. Thus, the optical absorption method allows to do prompt and effective monitoring the size of particles and size distribution in the course of the synthesis.

Our experiments demonstrated (curves 1 and 2 in Fig. 3) that particles of two sizes in colloidal solution exist for short synthesis time. Two peaks with 415 and $487 \mathrm{~nm}$ appear in the spectrum of the sample \#3, and 415 and $516 \mathrm{~nm}$ for the sample \#4. Here, the average sizes of particles in the sample \#3 are $\sim 1.2$ and $2.1 \mathrm{~nm}$, in the sample \#4 are $\sim 1.2$ and $2.6 \mathrm{~nm}$, correspondingly. Narrow peak at $415 \mathrm{~nm}$ corresponds to CdSe particles with smaller sizes, grown during the first stage of synthesis, which were detected in [3] as ultra-stable stoichiometric clusters $(\mathrm{CdSe})_{33}$ and $(\mathrm{CdSe})_{34}$. The peak at longer wavelengths corresponds to larger, definitely crystal-like, particles that grow at higher temperature. The dispersion of sizes of particles with the average size $2.1 \mathrm{~nm}$ is $12 \%$ (sample \#3) and $2.6 \mathrm{~nm}-19 \%$ (sample \#4). The presence of CdSe nanoparticles of both sizes in solution confirms our supposition that after nucleation only ultra-stable nanoclusters $(\mathrm{CdSe})_{33}$ and $(\mathrm{CdSe})_{34}$ were created, while the growth of larger particles occurs due to the Ostwald ripening, and the peak at $415 \mathrm{~nm}$ disappears gradually, which is demonstrated by the other curves for larger nanoparticles in Fig. 3.

Spectra of optical absorption and photoluminescence at room temperature for the samples $\# 1$ and $\# 2$ are presented in Fig. 4. In the optical absorption spectra, one peak located at 496 and $481 \mathrm{~nm}$ appears for the samples \#1 and \#2, correspondingly. The average size of particles is determined as $2.2 \mathrm{~nm}$ for the sample \#1, $\Delta d / d$ $=23 \%$, and $2.0 \mathrm{~nm}$ for the sample $\# 2, \Delta d / d=27 \%$. The sizes of particles for the sample \#1 obtained from optical measurements well correlate with the values obtained from the AFM measurements. For the sample \#2, the size of particles estimated from the optical measurements is larger than that obtained from AFM ones.

For the sample \#1, the excitonic luminescence is $25 \mathrm{~nm}$ shifted to the longer wavelength relatively to the absorption peak, for the sample \#2 the shift is $15 \mathrm{~nm}$. FWHM of luminescence spectra correlates with FWHM of absorption for both samples. 

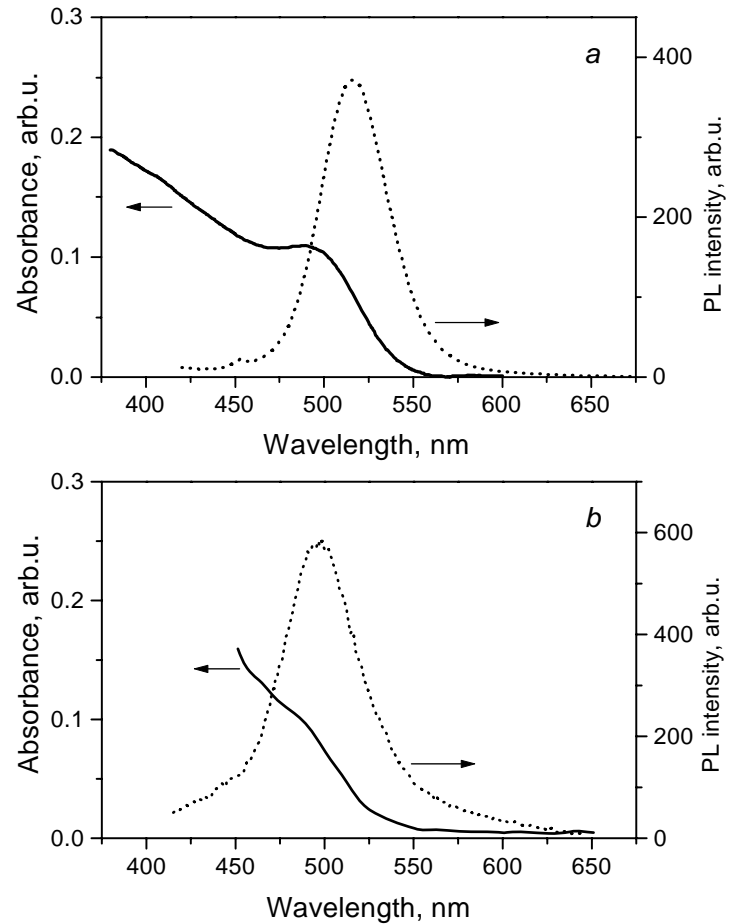

Fig. 4. Room temperature absorption and photoluminescence spectra of CdSe nanoparticles in toluene prepared with SNTA (sample \#1) (a), and Trilon B (sample \#2) (b). Excitation with $\mathrm{N}_{2}$ laser, $\lambda=337.1 \mathrm{~nm}$.

\section{Conclusions}

The modified reverse micelles method described in this work and based on the method [3] allows to obtain the CdSe nanoparticles in toluene solution in series of sizes with the average diameter from 1.2 to $3.2 \mathrm{~nm}$ and size distribution $\sim 12 \ldots 30 \%$. Covered by a shell of organic molecules, nanoparticles have crystal-like structure that determines their stability in toluene solution under absence of light and air for a long time. Simple empirical relation between the diameter of CdSe nanoparticles and exciton absorption wavelength is proposed, which allows to do prompt and effective monitoring the sizes of particles and size distribution during the synthesis. Optical absorption and photoluminescence measurements as well as EDX demonstrated good quality of obtained nanocrystallites. Besides, study of nanoparticles produced using two metal chelating agents (SNTA and Trilon B) revealed similar stoichiometric and optical properties. Trilon B is suitable for CdSe nanoparticles growth instead of SNTA. Because of higher stability of the complexes of Trilon $\mathrm{B}$ with $\mathrm{Cd}^{2+}$ ions, it is possible to use a higher temperature for growth, which allows preparation of larger size nanocrystals. Not less important is that using of Trilon B makes the CdSe nanoparticle synthesis cheaper.

\section{References}

1. P. Alivisatos, Perspectives on the physical chemistry of semiconductor nanocrystals // J. Phys. Chem. 100, p. 13226-13239 (1996).
2. B. Murray, D.J. Norris and M.G. Bawendi, Synthesis and characterization of nearly monodisperse $\mathrm{CdE}$ $(\mathrm{E}=\mathrm{S}, \mathrm{Se}, \mathrm{Te})$ semiconductor nanocrystallites // $J$. Amer. Chem. Soc. 115 (19), p. 8706-8715 (1993).

3. A. Kasuya, R. Sivamohan, Yu. Barnakov et al., Ultra-stable nanoparticles of CdSe revealed from mass spectrometry // Nature Materials 3 (2), p. 99103 (2004).

4. L. Xu, X. Huang, H. Huang et al., Surface modification and enhancement of luminescence due to quantum effects in coated $\mathrm{CdSe} / \mathrm{CuSe}$ semiconductor nanocrystals // Jpn J. Appl. Phys. 37, p. 3491-3494 (1998).

5. J. Zhu, X. Liao, X. Zhao and J. Wang, Photochemical synthesis and characterization of CdSe nanoparticles // Materials Letters 47, p. 339-343 (2001).

6. Y. Yan, Y. Li, X. Qian, J. Yin and Z. Zhu, Preparation and characterization of CdSe nanocrystals via $\mathrm{Na}_{2} \mathrm{SO}_{3}$-assisted photochemical route // Mater. Sci. and Eng. B 103, p. 202-206 (2003).

7. K.H. Yen, N.E. Stott, K.F. Jensen and M.G. Bawendi, A continuous-flow microcapillary reactor for the preparation of a size series of CdSe nanocrystals // Advanced Materials 15, p. 1858-1862 (2003).

8. M.J. Rosen, Surfactants and interfacial phenomena. Second Edition. John Willey \& Sons, NewYork, 1989.

9. M.-P. Pileni, The role of soft colloidal templates in controlling the size and shape of inorganic nanocrystals // Nature Materials 2(3), p. 145-150 (2003).

10. E. Lifshitz, I. Dag, I. Litvin, G. Hodes, S. Gorer, R. Reisfeld, M. Zelner and H. Minti, Optical properties of $\mathrm{CdSe}$ nanoparticle film prepared by chemical deposition and sol-gel method // Chem. Phys. Lett. 288, p. 188-196 (1988).

11. N. Chestnoy, T.D. Harris, R. Hull and L.E. Brus, Luminescence and photophysics of CdS semiconductor clusters: The nature of the emitting electronic state // J. Phys. Chem. 90, p. 3393-3399 (1986).

12. Y. Kayanuma, Quantum-size effects of interacting electrons and holes in semiconductor microcrystals with spherical shape // Phys. Rev. B 38 (14), p. 9797-9805 (1988).

13. Y. Wang and N. Herron, Quantum size effects on the exciton energy of CdS clusters // Phys. Rev. B 42 (11), p. 7253-7255 (1990).

14. Y. Wang, A. Suna, W. Mahler and R. Kasowski, $\mathrm{PbS}$ in polymers. From molecules to bulk solids // J. Chem. Phys. 87, p. 7315-7322 (1987).

15. K.B. Yatsimirskiy, V.P. Vasil'ev, Nonstability constants of complex compounds. Moscow, Publ. Academy of Science of USSR, 1959 (in Russian).

16. L.G. Sillen and A.E. Martell, Stability constants of metal-ion complexes. Second Edition. The Chemical Society, Burlington House, London, 1964.

17. I. Dmitruk, Yu. Barnakov and A. Kasuya, Laser photoetching in nanoparticles preparation and study of their physical properties // Low-dimensional systems: theory, preparation, and some applications. Kluwer Academic Publishers, p. 121-131 (2003). 